\title{
Birkenpollenextrakt im Plazebovergleich
}

\section{Die spezifische Immuntherapie ist indiziert bei IgE-vermittelter Sensibilisierung mit korrespondierenden klinischen Symptomen. Bisher gibt es aber nur eine begrenzte Anzahl von Studien, die den therapeutischen Nutzen und die Sicherheit bei Erwachsenen doppelblind und plazebokontrolliert dokumentieren.}

\begin{abstract}
n einer dänischen Studie erhielten 35
Patienten mit schwerer Rhinokonjunktivitis - einige von ihnen litten unter saisonalem allergischem Asthma über elf Monate regelmäßig subcutan entweder ein Cluster-Birkenpollen-Extrakt (Alutard SQ, Alk-Abellø) in steigender Dosis oder Plazebo-Injektionen.

In der aktiv behandelten Gruppe registrierte man einen signifikanten Rückgang der Symptome; parallel dazu benötigten die Behandelten weniger Medikamente, insbesondere Antihistaminika. Das generelle Wohlbefinden während der Pollensaison beurteilten
\end{abstract}

die Patienten aus der Verum-Gruppe stärker positiv als es die Reduktion von Symptomen und Medikamentenverbrauch vermuten ließ. Offenbar lässt sich der subjektive Gewinn an Lebensqualität, der mit der Immuntherapie erreichbar ist, mit einem objektivierbaren Symptomen-Score allein nicht fassen.

Das Auftreten von Nebenwirkungen unter der Depot-Immuntherapie bewegte sich auf Plazebo-Niveau.

\section{Fazit}

Auch unter den Bedingungen einer randomisierten, plazebokontrollierten

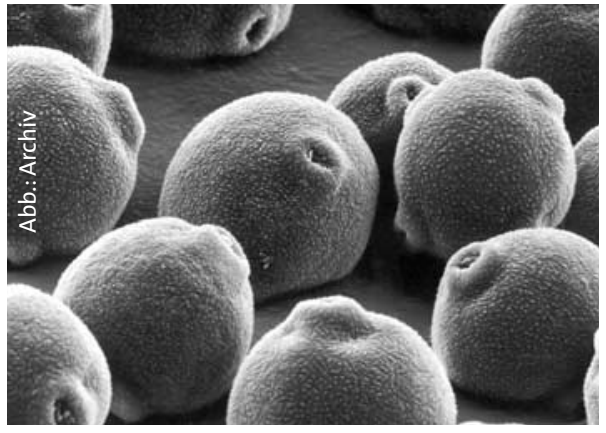

Birkenpollen unter dem Rasterelektronenmikroskop

Studie erwies sich die spezifische Immuntherapie mit subkutan verabreichtem Cluster-Birkenpollenpräparat als wirksame und sichere Behandlungsmöglichkeit bei schwerer IgE-vermittelter Rhinokonjunktivitis und Asthma durch Birkenpollen. $\quad w p a$

Bødtger $U$ et al: The safety and efficacy of subcutaneous birch pollen immunotherapy - a one-year, randomised, double-blind, placebo-controlled study. Allergy 2002; 57: 297-305

führen: Die Zahl der Studienabbrecher wegen ungenügender Krankheitskontrolle war in den Patientengruppen mit Zusatztherapie geringer. Studien, in denen die Effektivität einer zusätzlichen Gabe von Leukotrien-Rezeptorantagonisten mit der einer Verdoppelung der inhalativen Steroiddosis verglichen wurden, liegen bisher nicht vor.

\section{Fazit}

Die zusätzliche Gabe von LeukotrienRezeptorantagonisten $\mathrm{zu}$ inhalativen Kortikoiden kann die Asthmakontrolle leicht verbessern. Ob diese Therapiestrategie jedoch allgemein einer Dosissteigerung der Steroide vorzuziehen ist, ist derzeit noch offen. Bei gut eingestellten Patienten ist die zusätzliche Gabe nach einer Kortikoidreduktion aber offensichtlich sinnvoll.

$b k$

Ducharme FM. Anti-Leukotrienes as addon therapy to inhaled glucocorticoids in patients with asthma: systematic review of current evidence. BMJ 2002; 324: $1545-8$ 